\title{
Walking index for spinal cord injury (WISCI): an international multicenter validity and reliability study
}

\author{
JF Ditunno Jr*,1, PL Ditunno ${ }^{2}$, V Graziani ${ }^{1}, \mathrm{G} \mathrm{Scivoletto}^{3}, \mathrm{M}^{*}$ Bernardi $^{4}, \mathrm{~V}_{\text {Castellano }}{ }^{3}, \mathrm{M} \mathrm{Marchetti}^{4}, \mathrm{H} \mathrm{Barbeau}^{5}$, \\ HL Frankel ${ }^{6}, \mathrm{JM}^{\circ}$ 'Andrea Greve ${ }^{7}, \mathrm{H}-\mathrm{Y} \mathrm{Ko}^{8}, \mathrm{R}^{8}$ Marshall ${ }^{9}$ and P Nance ${ }^{10}$ \\ ${ }^{1}$ Department of Rehabilitation Medicine, Jefferson Medical College of Thomas Jefferson University, Philadelphia, \\ Pennsylvania USA; ${ }^{2}$ University of Sciences, Philadelphia, Pennsylvania USA; ${ }^{3}$ IRCCS Ospedale di Riabilitazione \\ Santa Lucia, Rome, Italy; ${ }^{4}$ Sapienza University, Rome, Italy; ${ }^{5}$ McGill University, Montreal, Canada; ${ }^{6}$ Stoke \\ Mandeville, Aylesbury, UK, ${ }^{7}$ University Hospital Sao Paulo, Sao Paulo, Brazil; ${ }^{8}$ Pusan National University, Pusan, \\ Korea; ${ }^{9}$ Royal Adelaide Hospital, Northfield, Australia; ${ }^{10}$ University of Manitoba, Manitoba, Canada
}

\begin{abstract}
Study design: Construction of an international walking scale by a modified Delphi technique. Objective: The purpose of the study was to develop a more precise walking scale for use in clinical trials of subjects with spinal cord injury (SCI) and to determine its validity and reliability.

Setting: Eight SCI centers in Australia, Brazil, Canada (2), Korea, Italy, the UK and the US. Methods: Original items were constructed by experts at two SCI centers (Italy and the US) and blindly ranked in an hierarchical order (pilot data). These items were compared to the Functional Independence Measure (FIM) for concurrent validity. Subsequent independent blind rank ordering of items was completed at all eight centers (24 individuals and eight teams). Final consensus on rank ordering was reached during an international meeting (face validation). A videotape comprised of 40 clips of patients walking was forwarded to all eight centers and inter-rater reliability data collected.

Results: Kendall coefficient of concordance for the pilot data was significant $(\mathrm{W}=0.843$, $P<0.001)$ indicating agreement among the experts in rank ordering of original items. FIM comparison (Spearman's rank correlation coefficient $=0.765, P<0.001$ ) showed a theoretical relationship, however a practical difference in what is measured by each scale. Kendall coefficient of concordance for the international blind hierarchical ranking showed significance $(\mathrm{W}=0.860, P<0.001)$ indicating agreement in rank ordering across all eight centers. Group consensus meeting resulted in a 19 item hierarchical rank ordered 'Walking Index for Spinal Cord Injury (WISCI)'. Inter-rater reliability scoring of the 40 video clips showed $100 \%$ agreement.

Conclusions: This is the first time a walking scale for SCI of this complexity has been developed and judged by an international group of experts. The WISCI showed good validity and reliability, but needs to be assessed in clinical settings for responsiveness.
\end{abstract}

Spinal Cord (2000) 38, 234-243

Keywords: spinal cord injury; walking index; disability evaluation; paraplegic classification

\section{Introduction}

Recovery of walking after paralysis of the muscles of the lower extremities, particularly following acute spinal cord injury is one of the primary goals of patients. In fact, Fraser ${ }^{1}$ reports that in patients with some preserved walking ability, improvements in walking quality were placed higher than bowel, bladder and sexual function.

*Correspondence: JF Ditunno Jr, Department of Rehabilitation Medicine, Regional Spinal Cord Injury Center of Delaware Valley, Thomas Jefferson University Hospital, Suite 375 Main Building, Philadelphia, PA 19107-5244, USA
Since $86 \%$ of patients with incomplete tetraplegia will recover some walking capacity, ${ }^{2}$ new treatments that are emerging may even further improve locomotion. The availability of new agents for neuroprotection, ${ }^{3,4}$ enhancement of neurological function, ${ }^{5-7}$ and, potentially, neuroregeneration ${ }^{8}$ as well as new training strategies all may result in the restoration of or improvement in walking following SCI. ${ }^{9-13}$ Thus a precise measure of walking that is able to be utilized in large clinical trials has become necessary. While some very precise measures of kinetics and kinematics by gait analysis and energy consumption ${ }^{14-16}$ do exist, these are often difficult to utilize in large trials. 
Recently a hierarchical scale of locomotor scores was developed for multicenter animal trials with high reliability, ${ }^{17}$ however a human counterpart is not currently available.

Recent multicenter studies ${ }^{4,18}$ have utilized the Functional Independence Measure (FIM) to determine ambulation status following acute spinal cord injury (SCI). The FIM, however, was developed as a measure to determine the burden of care or how much assistance a person with a disability requires ${ }^{19}$ and may have limitations as a measure of the functional limitation (capacity) for walking. It is of importance in drug trials $^{20}$ and in studies of the course of recovery ${ }^{2,21}$ following SCI, to document more precisely the improvement of impairment and corresponding functional limitation (capacity) irrespective of the burden of care. In addition, more research is needed ${ }^{22}$ not only in regard to refining the impairment and functional limitation measures, but in linking the phases between impairment and functional limitation (capacity).

It is for these reasons a new index is proposed for development, which will incorporate gradations of physical assistance and devices required for walking following paralysis of the lower extremities secondary to SCI. This should allow a more precise quantification of improvements in functional limitations secondary to improvements in the impairment. The first purpose of this study was to develop a more precise international walking scale by integrating walking aids, braces and physical assistance into a hierarchical order for use in clinical trials of subjects with SCI. The second purpose was to determine concurrent and face validity of the walking scale and its reliability.

\section{Methods}

Scale construction utilized a modified Delphi technique. ${ }^{23}$ The Delphi technique is a method for generating group consensus without having to have prolonged face-to-face interaction. An abbreviated version of the original technique involves four steps:

(1) Collection of participant's ideas

(2) Synthesis of list of ideas by each participant

(3) Integration of synthesis list by one person

(4) Rank in order or rating of the ideas by the participants until agreement occurs.

The WISCI scale was developed utilizing a modified Delphi technique as this procedure allows responses to be communicated via the mails (in this case electronic mail) and allows a large number of people from many geographical locations to participate. Phases of methodology are described below:

\section{Phase 1: Item Construction}

A twenty (20) item walking scale was developed by two clinical experts in spinal cord injury (after review of the literature and consultation with colleagues) These items were constructed to reflect levels of walking for spinal cord patients, combining the use of devices (parallel bars, walker, crutches and canes), braces and physical assistance (two persons, one person, or no assistance) for a distance of $10 \mathrm{~m}$ or $32.8 \mathrm{ft}$. Ten meters was chosen as a standardized walking distance which represents an international metric scale most frequently used throughout the world and often cited in the literature as a household distance, ${ }^{24}$ representing two lengths of standard parallel bars. The use of only this household distance does not include community distance. The scale does not take into consideration two additional extremely important parameters: walking speed and energy consumption. These critical dimensions of function (distance, speed and energy costs) can be studied and based on a valid hierarchy of severity in the future. For initial scale development, the following standardized definitions were used:

Physical assistance: 'Physical assistance of two persons' is moderate to maximum assistance; 'Physical assistance of one person' is minimal assistance

Braces: 'Braces' means one or two braces, either short or long leg; 'No braces' means no braces on either leg

Walker: 'Walker' is a conventional rigid walker without wheels

Crutches: 'Crutches' can be Lofstrand (canadian) or axillary

Cane: 'Cane' is a conventional straight cane

Original 20 Items: Walking Index for Spinal Cord Injury (WISCI):

Ambulates in parallel bars, with braces and physical assistance of two persons, less than the indicated $10 \mathrm{~m} .(/ / \mathrm{B} 210)$

Ambulates in parallel bars, with braces and physical assistance of two persons, $10 \mathrm{~m}$. (//B2)

Ambulates in parallel bars, with braces and physical assistance of one person, $10 \mathrm{~m}$. (//B1)

Ambulates in parallel bars, with braces and no physical assistance, $10 \mathrm{~m}$. (//B0)

Ambulates in parallel bars, no braces and no physical assistance, $10 \mathrm{~m}$. (//NB0)

Ambulates in parallel bars, no braces and physical assistance of one person, $10 \mathrm{~m}$. (//NB1)

Ambulates with walker, with braces and physical assistance of one person, $10 \mathrm{~m}$. (WB1)

Ambulates with walker, with braces and no physical assistance, $10 \mathrm{~m}$. (WB0)

Ambulates with walker, no braces and no physical assistance, $10 \mathrm{~m}$. (WNB0)

Ambulates with walker, no braces and physical assistance of one person, $10 \mathrm{~m}$. (WNB1)

Ambulates with two crutches, with braces and physical assistance of one person, $10 \mathrm{~m}$. (2CB1) 
Ambulates with two crutches, with braces and no physical assistance, $10 \mathrm{~m}$. (2CB0)

Ambulates with two crutches, no braces and no physical assistance, $10 \mathrm{~m}$. (2CNB0)

Ambulates with two crutches, no braces and physical assistance of one person, $10 \mathrm{~m}$. (2CNB1)

Ambulates with one cane/crutch, with braces and physical assistance of one person, $10 \mathrm{~m}$. (1CB1)

Ambulates with one cane/crutch, with braces and no physical assistance, $10 \mathrm{~m}$. (1CB0)

Ambulates with one cane/crutch, no braces and no physical assistance, $10 \mathrm{~m}$. (1CNB0)

Ambulates with one cane/crutch, no braces and physical assistance of one person, $10 \mathrm{~m}$. (1CNB1)

Ambulates with no devices, no braces and physical assistance of one person, $10 \mathrm{~m}$. (NDNB1)

Ambulates with no devices, no braces and no physical assistance, $10 \mathrm{~m}$. (NDNB0)

Abbreviations in parentheses above are as follows: (//indicates parallel bars); (W indicates walker); (C indicates crutches or cane); ( $\mathrm{B}$ indicates braces); (NB indicates no braces); (\# 1 or 2 indicates number of crutches or canes, or number of persons giving physical assistance); (ND indicates no devices).

\section{Phase 2: Blind Hierarchical Rank Ordering (Pilot Data)}

These 20 items were then presented in differing random orders to nine professionals working with spinal cord patients (two physician basic science researchers who specialize in human spinal cord gait research, three clinical researchers who specialize in spinal cord research, two clinical physicians and two physical therapists), at two independent spinal cord rehabilitation centers (one in Italy and one in the US). Each professional was asked to rank the items in order of severity of walking limitation secondary to the impairment, from most impaired to least impaired, and forward their data to the lead center.

\section{Phase 3: FIM Comparison for Concurrent Validity Check}

Subsequently the WISCI 20 items were presented in differing random orders to the same nine professionals and each was asked to assign a FIM score from the seven levels of the FIM locomotion domain to each WISCI item, and forward their data to the lead center.

\section{Phase 4: International Face Validation/Blind Hierarchical Rank Ordering}

The 20 items were then presented in differing random orders to experts at eight international spinal cord injury centers. This expert from each center was asked to independently rank the items in order of severity of walking limitation secondary to the impairment, from most impaired to least impaired, and to recruit two additional spinal cord professionals, preferably, a clinical physician and a physical therapist, who also ranked the items independently. Results were forwarded by electronic media to the lead center. The three individuals at each center were then asked to rank the items as a group and forward their consensus ranking via electronic media to the lead center. The instructions to international experts were as follows:

Step 1: The attached 'WISCI' scale items are presented in random order and should be independently, concurrently rank ordered (with no discussion) by three individuals at your center:

(1) one expert: clinical/investigator

(2) one physician who works with SCI patients

(3) one physical therapist who works with SCI patients

Step 2: These three independent rank orderings need to be identified by code and forwarded to the lead center.

Step 3: After the independent rank orderings are forwarded to the lead center, the three individuals become a team and rank order the items as a group, then forward this consensus rank ordering to the lead center.

\section{Phase 5: Statistical Analysis/Synthesis of Information} SPSS (Version 9.0) was utilized for all statistical analyses. For Phase 2, the Blind Hierarchical Ranking (Pilot Data), means and median were calculated for nine judges and the Kendall coefficient of concordance was calculated on the group data set. (The Kendall coefficient of concordance expresses the degree of agreement among several sets of rankings.) For Phase 3, the FIM Comparison for Concurrent Validity Check, Spearman's rank correlation coefficient was calculated on the WISCI item rank means and FIM score means across nine professionals and the frequency distribution described. (Spearman's rank correlation coefficient expresses the agreement between pairs of rankings.) For Phase 4, the International Face Validation/Blind Hierarchical Rank Ordering, individual (24 judges) and team (eight teams) means and median analyses were performed. The Kendall coefficient of concordance was calculated on the individual data set (24 judges) and on the team data set (eight teams). In addition, Spearman's rank correlation coefficients were calculated between all sub-group possible pairs of rankings:

between WISCI means for expert: clinical/investigator, and clinical physician

between WISCI means for expert: clinical/investigator, and physical therapist

between WISCI means for clinical physician and physical therapist 
Phase 6: Group Consensus Meeting for International Face Validation

Results of mean and median analyses were presented to the same international experts and the group was asked to come to a clinical judgment consensus areas of discrepancy generated by statistical analysis (discrepancy between means and median analysis rank ordering).

\section{Phase 7: International Reliability Study}

A videotape of a representative group of patients walking was circulated to the expert at the eight international centers for inter-rater reliability. The video taping represented 40 video segments in randomized order with three segments for some of the WISCI items; two segments for some of the WISCI items, and one segment for some of the WISCI items.

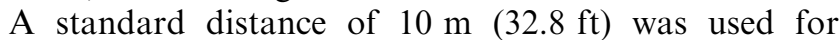
consistency; walking was continuous in one direction except in the parallel bars which required two lengths of $5 \mathrm{~m}$ with the patient turning once. Each expert was asked to view the videotape and independently score each segment according to the WISCI index. Each expert was to recruit the same spinal cord professionals that participated in Phase 4 and request independent scorings. The three independent scorings were forwarded to the lead center, subsequently the three individuals scored the video segments as a group. The inter-rater reliability instructions to international experts were as follows:

Step 1: The enclosed videotape is to be viewed for this part of the study. The tape is $46-$
$47 \mathrm{~min}$, and presents 40 randomized video segments of the spinal cord injured patients walking. Each segment is to be scored according to the WISCI index. The video segments need to be independently, concurrently scored (with no discussion) by the same three individuals who participated in the blind hierarchical rankings.

(1) one expert: clinical/investigator

(2) one physician who works with SCI patients

(3) one physical therapist who works with SCI patients

Step 2: These three independent scorings need to be identified by code and forwarded to the lead center.

Step 3: After the independent scorings are forwarded to the lead center, the three individuals become a team and score the video segments as a group, then forward this consensus scoring to the lead center.

\section{Results}

Phase 2: Blind Hierarchical Ranking (Pilot Data)

Pilot data means and median analysis with corresponding rank order are reported in Table 1. The Kendall coefficient of concordance (W) for the pilot data set $=0.843 ; \quad \mathrm{X}^{2}=144.073 ; \quad P<0.001$ for $\mathrm{df}=19$. (For Kendall coefficient of concordance when $\mathrm{N}>7$ use $\mathrm{X}^{2}$ for significance ${ }^{25}$ ).

Table 1 Pilot data means and median analysis rank ordering

\begin{tabular}{|c|c|c|c|c|}
\hline WISCI items (see text for item code) & Mean & $\begin{array}{c}\text { Rank order } \\
\text { based on mean }\end{array}$ & Median & $\begin{array}{c}\text { Rank order } \\
\text { based on median }\end{array}$ \\
\hline$/ / \mathrm{B} 2<10$ & 1 & 1 & 1 & 1 \\
\hline$/ / \mathrm{B} 2$ & 2 & 2 & 2 & 2 \\
\hline$/ / \mathrm{B} 1$ & 3 & 3 & 3 & 3 \\
\hline$/ / \mathrm{B} 0$ & 6.22 & 5 & 5 & 5 \\
\hline$/ / \mathrm{NB} 0$ & 10.67 & 10 & 12 & 13 \\
\hline$/ / \mathrm{NB} 1$ & 6.89 & 6 & 7 & 7 \\
\hline WB1 & 5.44 & 4 & 5 & 5 \\
\hline WB0 & 9.33 & 8 & 9 & 9 \\
\hline WNB0 & 13.33 & 14 & 14 & 14 \\
\hline WNB1 & 9.44 & 9 & 8 & 8 \\
\hline $2 \mathrm{CB} 1$ & 7.67 & 7 & 7 & 7 \\
\hline $2 \mathrm{CB} 0$ & 11.11 & 12 & 11 & 10 \\
\hline $2 \mathrm{CNB} 0$ & 15.44 & 17 & 15 & 15 \\
\hline 2CNB1 & 11.78 & 13 & 12 & 13 \\
\hline 1CB1 & 11 & 11 & 12 & 13 \\
\hline 1CB0 & 14.89 & 15 & 16 & 17 \\
\hline $1 \mathrm{CNB} 0$ & 18.56 & 19 & 19 & 19 \\
\hline $1 \mathrm{CNB} 1$ & 15.11 & 16 & 16 & 17 \\
\hline NDNB1 & 17.11 & 18 & 18 & 18 \\
\hline NDNB0 & 20 & 20 & 20 & 20 \\
\hline
\end{tabular}


Phase 3: FIM Comparison for Concurrent Validity Check

Spearman's rank correlation coefficient comparing WISCI item rank means and FIM score means across nine professionals $=0.765$. The frequency distribution indicates that $80 \%$ of the WISCI items fell into two of the FIM categories (Figure 1).

Phase 4: International Face Validation/Blind Hierarchical Rank Ordering

International individual (24 judges) and team (eight teams) means and median analysis with corresponding

\section{WISCI/FIM}

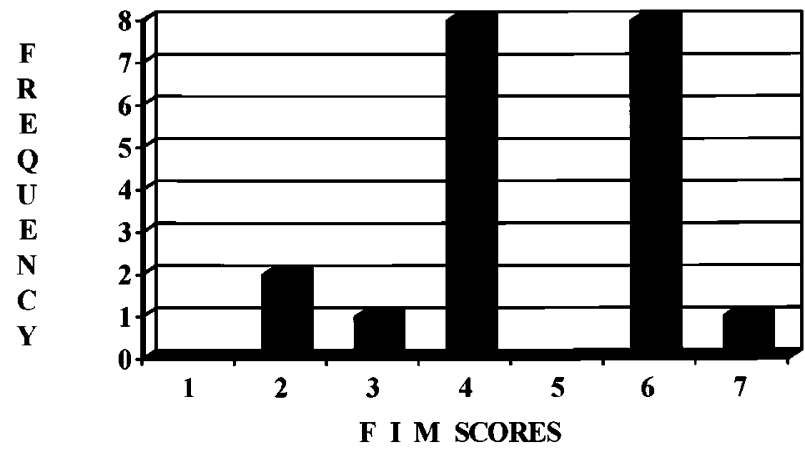

Figure 1 Frequency distribution of WISCI items scored on FIM rank order are reported in Table 2. Twelve items were consistent in rank order across all analyses. For eight items (representing three clusters of discrepancy) rank orderings were less clear. The Kendall coefficient of concordance $(\mathrm{W})$ for the international individual data set $=0.860 ; \mathrm{X}^{2}=392.107 ; P<0.001$ for $\mathrm{df}=19$; and for the team data set $=0.872 ; X^{2}=132.529 ; P<0.001$ for $\mathrm{df}=19$. Spearman's rank correlation coefficients were calculated between all sub-group possible pairs of rankings and are reported in Table 3.

\section{Phase 6: Group Consensus Meeting for International} Face Validation

For the eight items (representing three clusters of discrepancy between means and median analysis rank ordering) the group conceptually agreed the following.

(1) For the triad rank orders 4,5,6 the clinical consensus was that using a walker is less impaired than parallel bars, therefore specified the order for this triad based on median analysis: rank order 4 (//NB1); rank order $5(/ / \mathrm{B} 0)$; rank order $6(\mathrm{WB} 1)$.

(2) For the triad rank orders 10, 11, 12, item $10(/ /$ NB0) had a standard deviation $>3.0$. The clinical consensus was to eliminate item 10 because of this unacceptable variance and the clinical impression that this level would be seen very infrequently. In addition, the clinical consensus was that using braces, irrespective of one or two canes, reflects a more severely impaired individual than someone with no braces, therefore, adopt the item (1CB1) as more impaired than (2CNB1).

Table 2 International individual (24 judges) and team (eight teams) means and median analysis rank ordering

\begin{tabular}{|c|c|c|c|c|c|c|c|c|}
\hline $\begin{array}{l}\text { WISCI items } \\
\text { (see text for item code) }\end{array}$ & $\begin{array}{l}\text { Individual } \\
\text { means }\end{array}$ & $\begin{array}{l}\text { Rank order } \\
\text { based on } \\
\text { individual } \\
\text { mean }\end{array}$ & $\begin{array}{l}\text { Individual } \\
\text { medians }\end{array}$ & $\begin{array}{l}\text { Rank order } \\
\text { based on } \\
\text { individual } \\
\text { median }\end{array}$ & $\begin{array}{l}\text { Team } \\
\text { means }\end{array}$ & $\begin{array}{l}\text { Rank order } \\
\text { based on } \\
\text { team means }\end{array}$ & $\begin{array}{l}\text { Team } \\
\text { medians }\end{array}$ & $\begin{array}{c}\text { Rank order } \\
\text { based on } \\
\text { team } \\
\text { medians }\end{array}$ \\
\hline$/ / \mathrm{B} 2<10$ & 1 & 1 & 1 & 1 & 1 & 1 & 1 & 1 \\
\hline$/ / \mathrm{B} 2$ & 2 & 2 & 2 & 2 & 2 & 2 & 2 & 2 \\
\hline$/ / \mathrm{B} 1$ & 3.04 & 3 & 3 & 3 & 3 & 3 & 3 & 3 \\
\hline$/ / \mathrm{B} 0$ & 6.21 & 6 & 5 & 5 & 6 & 6 & 5 & 5.5 \\
\hline$/ / \mathrm{NB} 0$ & 10.04 & 10 & 10.5 & 10 & 10.25 & 10 & 11 & 11 \\
\hline$/ / \mathrm{NB} 1$ & 5.83 & 4.5 & 4 & 4 & 5.88 & 5 & 4.5 & 4 \\
\hline WB1 & 5.83 & 4.5 & 5.5 & 6 & 5.50 & 4 & 5 & 5.5 \\
\hline WB0 & 9.33 & 9 & 9 & 9 & 9.63 & 9 & 9.5 & 9 \\
\hline WNB0 & 13.16 & 14 & 14 & 14 & 13.88 & 14 & 14.5 & 14 \\
\hline WNB1 & 9.08 & 8 & 8 & 8 & 9.25 & 8 & 9 & 8 \\
\hline 2CB1 & 8 & 7 & 7 & 7 & 7.75 & 7 & 7 & 7 \\
\hline 2CB0 & 12.04 & 13 & 13 & 13 & 11.75 & 13 & 12.5 & 13 \\
\hline $2 \mathrm{CNB} 0$ & 15.58 & 17 & 16 & 16.5 & 15.88 & 17 & 16 & 16 \\
\hline $2 \mathrm{CNB} 1$ & 11.17 & 11 & 12 & 11.5 & 11.63 & 12 & 11.5 & 12 \\
\hline $1 \mathrm{CB} 1$ & 11.71 & 12 & 12 & 11.5 & 11.13 & 11 & 10.5 & 10 \\
\hline $1 \mathrm{CB} 0$ & 15.25 & 16 & 16 & 16.5 & 15.25 & 16 & 16.5 & 17 \\
\hline 1CNB0 & 18.58 & 19 & 19 & 19 & 18.63 & 19 & 19 & 19 \\
\hline 1CNB1 & 14.54 & 15 & 15.5 & 15 & 14.13 & 15 & 15 & 15 \\
\hline NDNB1 & 17.58 & 18 & 18 & 18 & 17.50 & 18 & 18 & 18 \\
\hline NDNB0 & 20 & 20 & 20 & 20 & 20 & 20 & 20 & 20 \\
\hline
\end{tabular}


Table 3 Spearman's rho for sub-group pairs of rankings $(* P<0.01)$

\begin{tabular}{lcc}
\hline & $\begin{array}{c}\text { Spinal cord } \\
\text { injury expert }\end{array}$ & $\begin{array}{c}\text { Clinical } \\
\text { physician }\end{array}$ \\
\hline $\begin{array}{l}\text { Spinal cord injury expert } \\
\text { Clinical physician }\end{array}$ & $0.968^{*}$ & \\
Physical therapist & $0.944^{*}$ & $0.974^{*}$ \\
\hline
\end{tabular}

(3) For the pair rank orders 16, 17 the clinical consensus was that using braces, irrespective of one or two canes, reflects a more severely impaired individual than someone with no braces, therefore, adopt the item (ICB0) as more impaired than (2CNB0).

(4) The 19 item hierarchical rank ordered scale was adopted (see Appendices A and B)

\section{Phase 7: International Reliability Study}

Inter-rater scoring of the 40 video clips using the WISCI scale was in perfect agreement across all 24 individual international participants and across all eight teams therefore, inter-rater reliability $=100 \%$.

\section{Discussion}

This study was carried out for two purposes. The first purpose was to add greater precision to an international walking scale by integrating walking aids, braces and physical assistance into a hierarchical order for use in clinical trials of subjects with SCI. The second purpose was to assess validity (face and concurrent) and reliability of the scale.

We have demonstrated that the WISCI is a more precise scale than the FIM for documenting change in levels of walking based on Phase 3 analysis, the WISCI/FIM comparison. The FIM is a reliable and valid measurement tool for burden of care, but it was not originally developed to be used to document change in levels of walking. As such it places greater emphasis on (or is driven by) physical assistance with scores of maximum assistance (2), moderate assistance (3), minimal assistance (4), supervision (5), no helper (6 and 7). The FIM does not distinguish among devices used for independent movement, and does not integrate use of devices within levels of physical assistance. The frequency distribution (Figure 1) does reflect a difference in scale range structure; ${ }^{24}$ the original WISCI had 20 levels in comparison to the FIM which has seven levels. This difference in scale range does not fully explain why $80 \%$ of the original WISCI items fall into only two FIM categories. One possibility is that the WISCI is conceptually driven more by devices, and less by physical assistance. In fact, the WISCI assigns differences in level with an emphasis on devices for all items except two (NDNB1, NDNB0), where no devices are used. For example, once the patient's level is out of the parallel bars, the only physical assistance stated in the items is 'physical assistance of one person' (comparable to FIM scale 4) or 'no physical assistance' (comparable to FIM scale 6). It was the clinical judgment of the nine professionals that a distinction between levels of physical assistance integrated with devices betterreflected improvement in walking than gradations of physical assistance alone. To further ensure clinical concurrent validation against the FIM, however, a group of patients will be evaluated by the WISCI and separately on the FIM in clinical trials which are in process.

Validity and reliability of the WISCI were demonstrated in Phases 2 through 7. Face validity was demonstrated in Phases 2,4 and 6. Analysis of the Phase 2 (Blind Hierarchical Ranking of Pilot Data) suggests that a scale premised on hierarchical rankings can show consensus among SCI specialists. The Kendall coefficient of concordance, which gives an index of the relation (agreement) among several rankings of items, was significant, suggesting that all of the nine judges participating in the pilot phase were applying essentially the same standard in ranking the items. Phase 4 (International Face Validation/Blind Hierarchical Ranking ordering), and Phase 6 (Group Consensus Meeting for International Face Validation) both address international face validation, and show that in fact consensus was achieved at an international level. Kendall's coefficients of concordance (index of agreement) for both the international individual data set and the international team data set were significant suggesting that all the international participants were applying essentially the same standard when ranking the items. In addition Spearman's rank correlation coefficients show the strength of agreement between all professional sub-group rankings; all of these correlations are highly significant giving a second measure that supports agreement in rank ordering. Twelve of the items show consistent assignment of rank order across all participants. For eight of the items (representing three clusters) there was discrepancy between the means and median analysis rank orderings. A face to face open-discussion consensus meeting (Phase 6) resolved these discrepancies based on clinical judgment. Therefore, results of Phases 2,4 and 6 indicate that the WISCI has good face validity across an international group of judges. In addition, there is significant agreement across all judges that the hierarchical rank ordering represents a measurable change in walking along a dimension of most impaired to least impaired.

Concurrent validity, which was demonstrated in Phase 3 (FIM Comparison for Concurrent Validity Check) shows that there is overlap between the domains measured by the WISCI and the FIM locomotion scores (Spearman rho $=0.765$, in variance terms this can be expressed as explaining $59 \%$ of the variance). This indicates that both measures address the same overall theoretical concept, mobility, and gives a measure of concurrent validity. The implication 
is that while there is overlap on a conceptual level, there is a practical difference in what is being measured by each scale. The WISCI is a scale of walking limitation secondary to impairment while the FIM focuses on burden of care and locomotion in the environment.

Reliability is addressed in Phase 7 (International Reliability Study). Analysis of international individual and international team data shows $100 \%$ agreement for the assignment of WISCI scores to all 40 video clips. Each of the 24 raters assigned the same WISCI score to each of the 40 video clips; and team assignment was the same. This shows excellent interrater reliability and strengthens the psychometric properties of the scale. Inter-rater reliability using the 40 video segments needs to be performed by naïve experts who were not involved in the scale development, and real time inter-rater reliability needs to be included in future clinical trials.

Thus, we have a new index for walking following SCI that shows good validity and reliability. The past use of scales for measuring walking in SCI trials has been very limited. The first scale to measure walking in SCI was the Frankel scale, ${ }^{26}$ in which the Frankel D level (functional muscles) indicated the subjects recovered walking function. The frequent use of this scale as an outcome measure, however, was for purposes of determining severity of injury and not walking. In fact, Maynard ${ }^{27}$ suggested Frankel D be divided into subjects who walk and do not walk, and Benzel ${ }^{28}$ subsequently developed a modified Frankel Scale to 7 levels, which added three levels of walking. Although, the Benzel scale was utilized recently in a large trial in which an improvement of two levels was the primary endpoint, it has not been studied as to its psychometric properties. The WISCI has 19 levels of walking and is unique because it integrates the use of devices, both for the arms and legs and physical assistance into a hierarchical order. Arm devices for walking include the parallel bars, walkers, crutches and canes, while leg devices include short and long leg braces. The integration of devices and physical assistance to measure walking goes beyond an impairment classification limited to muscle weakness and falls into the category of functional limitation (capacity).

Functional limitation (or capacity) is included in the disablement model of the National Center for Medical Rehabilitation Research, ${ }^{29}$ and the enablement model of the Institute of Medicine, ${ }^{22}$ but is not included in the former World Health Organization model, ${ }^{30}$ which defines walking disability as ambulation on level terrain. The proposed 1997 World Health Organization International Classification of Impairments, Activities and Participation (ICIDH-2) ${ }^{31}$ lists walking as an activity on a person level and is similar to the Institute of Medicine classification of functional limitation. Functional limitation refers to the whole person and is a phase between weakness of muscles (impairment at the organ level) and disability, which is the function of the individual in the environment. In order to describe walking in the whole person in regard to functional limitation, consideration must be given to the role of training and substituted function. For example a patient with completely paralyzed lower extremities may show 'recovery of walking' with no increase in strength of the lower extremities. It would appear a patient with complete paraplegia could achieve levels $1-4,6-8$, and possibly 11 based solely on training. Patients with incomplete SCI however, could possibly transition through all 19 levels as a result of improvement in strength and/or training. Since walking falls more in the category of limitations at the whole person level, it should be regarded as a functional limitation rather than a disability.

Disability scales that measure mobility in the environment are often used in clinical trials to measure ambulation. The FIM has already been described as a measure of burden of care or amount of physical assistance, rather than gradations of use of arm and leg devices. A recent disability scale, the Spinal Cord Independence Measure ${ }^{24}$ has been offered as an improvement over the FIM in regard to precision, with good reliability but validation limited to the authors. Another general disability scale, the Clinical Outcome Variable, ${ }^{32}$ measures walking very precisely in regard to distances and time with good reliability, but unpublished validation. Neither scale completely integrates arm devices, leg devices and physical assistance in a hierarchical scale. The WISCI is unique in that it is a functional capacity (limitation) scale that is based on hierarchical ranking and integrates both gradations of physical assistance and devices. The development process of the WISCI is also unique in that a modified Delphi technique was used and an international group served as judges.

Thus, the purpose of the current WISCI scale for use in clinical trials is to document changes in functional capacity for a distance of $10 \mathrm{~m}$ in a rehabilitation setting. It is anticipated that the greater precision achieved by the hierarchical ranking will be more sensitive to change as the result of drug, training or other interventions. For immediate use in clinical trials, however, the scale requires the addition of more detailed descriptors of the use of devices (arm and leg), physical assistance and comfort of the subject in order to ensure accurate interpretation by observers, and to make proper modifications of the hierarchy in the future. A format to be used for descriptors in the interpretation and scoring in clinical trials is listed (see Appendices A and B). The current WISCI scale, which is limited to documentation of functional capacity, will also require further validation to measure function as related to a disability. Different distances (household and community), velocity and energy requirements will need to be added.

Thus, this scale, which links impairment to functional capacity, needs to be further linked to a disability measure. ${ }^{22}$ In linking functional capacity to a disability measure, consumer preference will also be important. The validation of disability scales by 
objective parameters, however, has been limited. Objective measures, such as the vertical forces of the arms used in walking with devices, have been reported $^{33}$ to support the hierarchical ranking by the experts in this study and show a decreasing gradation of force required for use of walkers, crutches and canes, respectively. The peak forces utilized by the arms of spinal cord injured subjects in crutch walking correlated with the physiologic cost of walking in another study. ${ }^{34}$ Future research in the correlation of force and energy costs may be of value in the further validation. Walking behavior, however, involves many factors such as weakness, postural problems related to weight bearing, balance, coordination, propulsion, sensation, hyperactivity, all which 'may interfere with coordination, balance and other factors important to walking. ${ }^{35}$ A scale that will be useful in multicenter clinical trials, however, must be valid, reliable and responsive to change, but also practical. Therefore, a quantitative measure needs to be selected that can be used across various sites.

For these reasons current and future research is focused on clinical validation and responsiveness of the scale and retrospective and prospective studies are in progress.

\section{Acknowledgements}

This study was supported in part by the Regional Spinal Cord Injury Center of Delaware Valley; Model Sci systems Grant H133N950021 to Thomas Jefferson University from the National Institute on Disability and Rehabilitation Research, and by a grant from: IRCCS Ospedale di Riabilitazione I.R.C.C.S. Santa Lucia, Rome Italy. The authors gratefully acknowledge the help of Bruce $\mathrm{H}$ Dobkin, MD, (University of California, Los Angeles, CA). Robert L Waters, MD (Rancho Los Amigos Medical Center, Downey, CA) and Michelle Basso, EdD, PT (Ohio State University Health Science Center, Columbus, $\mathrm{OH}$ ) for review of the manuscript.

\section{References}

1 Fraser MH. 1999; Personal Communication.

2 Burns SP et al. Recovery of ambulation in motor-incomplete tetraplegia. Arch Phys Med Rehabil 1997; 78: 1169-1172.

3 Bracken MB et al. A randomized, controlled trial of methylprednisolone or naloxone in the treatment of acute spinal-cord injury. Results of the Second National Acute Spinal Cord Injury Study. N Eng J Med 1990; 322: $1405-1411$.

4 Bracken MB et al. Administration of methylprednisolone for 24 or 48 hours or tirilazad mesylate for 48 hours in the treatment of acute spinal cord injury. Results of the Third National Acute Spinal Cord Injury Randomized Controlled Trial. National Acute Spinal Cord Injury Study. JAMA 1997; 277: 1597- 1604.

5 Hansebout RR, Blight AR, Fawcett S, Reddy K. 4-Aminopyridine in chronic spinal cord injury: a controlled, double-blind, crossover study in eight patients. J Neurotrauma 1993; 10: $1-18$.

6 Hayes KC et al. Preclinical trial of 4-aminopyridine in patients with chronic spinal cord injury. Paraplegia 1993; 31: 216-224.

7 Hayes KC et al. 4-Aminopyridine-sensitive neurologic deficits in patients with spinal cord injury. J Neurotrauma 1994; 11: 433 446.
8 Cheng $\mathrm{H}$, Olson L. A new surgical technique that allows proximodistal regeneration of 5-HT fibers after complete transection of the rat spinal cord. Exp Neurol 1995; 136: 149161.

9 Rossignol S, Barbeau H. Pharmacology of locomotion: an account of studies in spinal cats and spinal cord injured subjects. J Am Paraplegia Soc 1993; 16: 190- 196.

10 Norman KE, Pepin A, Ladouceur M, Barbeau H. A treadmill apparatus and harness support for evaluation and rehabilitation of gait. Arch Phys Med Rehabil 1995; 76: $772-778$.

11 Ladouceur M, Pepin A, Norman KE, Barbeau H. Recovery of walking after spinal cord injury. Adv Neurol 1997; 72: 249-255.

12 Harkema SJ et al. Human lumbosacral spinal cord interprets loading during stepping. J Neurophysiol 1997; 77: 797-811.

13 Dietz V, Wirz M, Jensen L. Locomotion in patients with spinal cord injuries. Phys Ther 1997; 77: 508-516.

14 Waters RL, Yakura JS, Adkins R, Barnes G. Determinants of gait performance following spinal cord injury. Arch Phys Med Rehabil 1989; 70: $811-881$.

15 Bernardi $\mathrm{M}$ et al. Cost of walking and locomotor impairment. $J$ Electromyogr Kinesiol 1999; 9: 149-157.

16 Bernardi $\mathrm{M}$ et al. The efficiency of walking of paraplegic patients using a reciprocating gait orthosis. Paraplegia 1995; 33: $409-$ 415.

17 Basso DM et al. MASCIS evaluation of open field locomotor scores: effects of experience and teamwork on reliability. Multicenter Animal Spinal Cord Injury Study. J Neurotrauma 1996; 13: 343 - 359 .

18 Spinal cord injury: clinical outcomes from the Model Systems. Stover SL, DeLisa SA, Whiteneck CC (eds). Gaithersburg, MD Aspen Publishers, Inc., 1995.

19 Hamilton BB, Granger CV, Sherwin FS. A uniform national data system for medical rehabilitation. In: Fuhrer MJ, ed. Rehabilitation Outcomes: Analysis and Measurement. Baltimore, MD.: Paul H. Brookes, 1987: pp 137-147.

20 Reier PJ et al. Workshop on intraspinal transplantation and clinical application. J Neurotrauma 1994; 11: $369-377$.

21 Ditunno Jr JF, Stover SL, Freed MM, Ahn JH. Motor recovery of the upper extremities in traumatic quadriplegia: a multicenter study. Arch Phys Med Rehabil 1992; 73: 431-436.

22 Brandt EN, Pope AM. Enabling America: Assessing the role of rehabilitation science and engineering. Washington, DC: National Academy Press, 1997; pp 1-332.

23 Dalkey ND. In: Delphi. Chicago: Rand Corporation, 1967.

24 Catz A et al. Spinal cord independence measure - a new disability scale for patients with spinal cord lesions. Spinal Cord 1997; 35: $850-856$.

25 Siegel S. Nonparametric Statistics for the Behavioral Sciences. New York: McGraw-Hill, 1956.

26 Frankel HL et al. The value of postural reduction in the initial management of closed injuries of the spine with paraplegia and tetraplegia, Part 1. Paraplegia 1969; 7: 179-192.

27 Maynard FM et al. Neurological prognosis after traumatic quadriplegia. Three-year experience of California Regional Spinal Cord Injury Care System. J Neurosurg 1979; 50: 611-661.

28 Benzel EC, Larson SJ. Functional recovery after decompressive spine operation for cervical spine fractures. Neurosurgery 1987; 20: $742-746$

29 National Advisory Board on Medical Rehabilitation Research Plan for the National Center for Medical Rehabilitation Research. Bethesda, Maryland: National Institutes of Health, 1993.

30 World Health Organization. International Classification of Impairments, Disability and Handicaps: A Manual of Classification Relating to the Consequences of Disease. Geneva, Switzerland: World Health Organization, 1980.

31 World Health Organization. International Classification of Impairments. Activities and Participation: A Manual of Dimensions of Disablement and Functioning. Geneva, Switzerland: World Health Organization, 1997.

32 Seaby L, Torrance G. Reliability of a physiotherapy functional assessment used in a rehabilitation setting. Physiother Can 1989; 41: $264-271$. 
33 Melis EH, Torres-Moreno R, Barbeau H, Lemaire ED. Analysis of assisted-gait characteristics in persons with incomplete spinal cord injury. Spinal Cord 1999; 37: 430-439.

34 IJzerman MJ et al. Validity and reproducibility of crutch force and heart rate measurements to assess energy expenditure of paraplegic gait. Arch Phys Med Rehabil 1999; 80: 1017-1023.
35 Barbeau $\mathrm{H}$ et al. Walking after spinal cord injury: evaluation, treatment, and functional recovery. Arch Phys Med Rehabil 1999; 80: $225-235$.

\section{Appendix A}

\section{Walking Index for Spinal Cord Injury (WISCI)}

Physical limitation for walking secondary to impairment is defined at the person level and indicates the ability of a person to walk after spinal cord injury. The development of this assessment index required a rank ordering along a dimension of impairment, from the level of most severe impairment (1) to least severe impairment (19) based on the use of devices, braces and physical assistance of one or more persons. The order of the levels suggests each successive level is a less impaired level than the former. The ranking of severity is based on the severity of the impairment and not on functional independence in the environment. The following definitions standardize the terms used in each item:

Physical assistance: 'Physical assistance of two persons' is moderate to maximum assistance 'Physical assistance of one person' is minimal assistance.

Braces: $\quad$ 'Braces' means one or two braces, either short or long leg 'No braces' means no braces on either leg.

Walker: 'Walker' is a conventional rigid walker without wheels.

Crutches: 'Crutches' can be Lofstrand (canadian) or axillary

Cane: 'Cane' is a conventional straight cane.

\section{Level Description}

1 Ambulates in parallel bars, with braces and physical assistance of two persons, less than 10 meters.

2 Ambulates in parallel bars, with braces and physical assistance of two persons, 10 meters.

3 Ambulates in parallel bars, with braces and physical assistance of one person, 10 meters.

$4 \quad$ Ambulates in parallel bars, no braces and physical assistance of one person, 10 meters.

$5 \quad$ Ambulates in parallel bars, with braces and no physical assistance, 10 meters.

6 Ambulates with walker, with braces and physical assistance of one person, 10 meters.

7 Ambulates with two crutches, with braces and physical assistance of one person, 10 meters.

8 Ambulates with walker, no braces and physical assistance of one person, 10 meters.

9 Ambulates with walker, with braces and no physical assistance, 10 meters.

10 Ambulates with one cane/crutch, with braces and physical assistance of one person, 10 meters.

11 Ambulates with two crutches, no braces and physical assistance of one person, 10 meters.

12 Ambulates with two crutches, with braces and no physical assistance, 10 meters.

13 Ambulates with walker, no braces and no physical assistance, 10 meters.

14 Ambulates with one cane/crutch, no braces and physical assistance of one person, 10 meters.

15 Ambulates with one cane/crutch, with braces and no physical assistance, 10 meters.

16 Ambulates with two crutches, no braces and no physical assistance, 10 meters.

Ambulates with no devices, no braces and no physical assistance, 10 meters 


\section{Appendix B}

\section{WISCI Scoring Sheet}

Patient Name

Date

Check descriptors which apply to current walking performance, then assign the highest level of walking performance

In scoring a level, one should choose the level at which the patient is safe as judged by the therapist, with patient's comfort level described. If devices other than stated in the standard definitions are used, they should be documented as descriptors. If there is a discrepancy between two observers, the higher level should be chosen.

\section{Descriptors}

\begin{tabular}{llll}
\hline Devices & Braces & Assistance & Patient reported comfort level \\
\hline$/ /$ bars $<10 \mathrm{ft}$ & Long Leg Braces - Uses 2 & Max Assist x 2 people & Very comfortable \\
& & Uses 1
\end{tabular}

// bars $10 \mathrm{ft}$

Walker - Standard

Rolling Platform

Crutches - Uses 2 Uses 1

Canes - Quad

Uses 2

Uses 1

\section{Short Leg Braces - Uses 2} Uses 1

Min/Mod assist x 2 people

Min/Mod assist x 1 person
Slightly comfortable

Neither comfortable nor uncomfortable

Slightly uncomfortable

Very uncomfortable

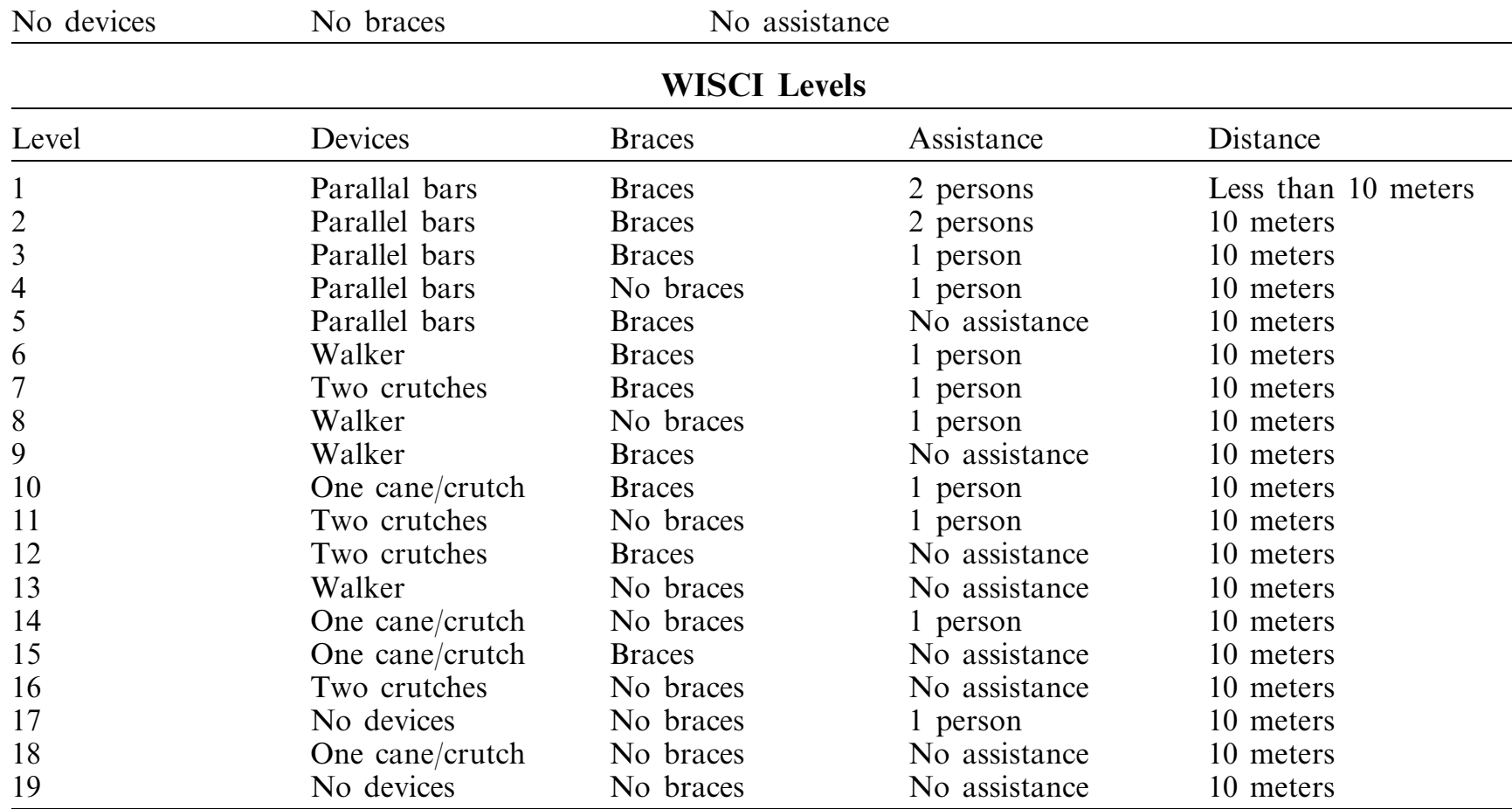

Level assigned 